\title{
Structure, catalytic activity and evolutionary relationships of 1-aminocyclopropane-1-carboxylate synthase, the key enzyme of ethylene synthesis in higher plants
}

\author{
Małgorzata Jakubowicz ${ }^{\bowtie}$
}

\section{Institute of Molecular Biology and Biotechnology, Adam Mickiewicz University, Poznań, Poland}

Received: 15 March, 2002; revised: 18 June, 2002; accepted: 01 September, 2002

Key words: 1-aminocyclopropane-1-carboxylate synthase, ethylene synthesis

\begin{abstract}
Both ethylene and the enzymes of ethylene synthesis are subjects of intensive scientific investigation. The present review discusses structure, catalytic activity and evolutionary relationships of 1-aminocyclopropane-1-carboxylate synthase, identified for the first time in ripening tomato in 1979. This enzyme is responsible for the conversion of $S$-adenosyl-L-methionine to 1-aminocyclopropane-1-carboxylic acid, which is the key step of ethylene synthesis in higher plants. The role of this enzyme (especially in the fruit ripening) was demonstrated in 1991 in transgenic tomato plants, expressing 1-aminocyclopropane-1-carboxylate synthase antisense RNA. On the basis of mutagenesis and crystallization of the enzyme, new data were provided on the three-dimensional structure and amino-acid residues which are critical for catalysis. The control of ethylene production is of great interest for plant biotechnology because it can delay senescence and overmaturation. These processes are responsible for large loss of vegetables and fruit on storage. Detailed structural and biochemical data are necessary to help design 1-aminocyclopropane-1-carboxylate synthase inhibitors, whose application is expected to have immense agricultural effects.
\end{abstract}

\footnotetext{
${ }^{\otimes}$ Address for correspondence: Małgorzata Jakubowicz: Institute of Molecular Biology and Biotechnology, Adam Mickiewicz University, Międzychodzka 5, 60-371 Poznań, Poland; phone/fax: (48 61) 8292730 , e-mail goja@amu.edu.pl

Abbreviations: ACC, 1-aminocyclopropane-1-carboxylic acid; ACS, 1-aminocyclopropane-1-carboxylate synthase; AVG, aminoethoxyvinylglycine; KMB, 2-keto-4-methylthiobutyrate; L-VG, L-vinylglycine; PMP, pyridoxalamine; MACC, 1-(malonyl-amino)cyclopropane-1-carboxylic acid; MTA, $5^{\prime}$-methylthioadenosine; MTR, 5'-methylthioribose; MVG, methoxyethoxyvinylglycine; PLP, pyridoxal 5'-phosphate; SAHC, $S$-adenosylhomocysteine; SAM, $S$-adenosyl-L-methionine; $3 \mathrm{dz}$-SAHC, 3-deazaadenosylhomocysteine; SIBA, $S$-isobutyladenosine; 1-dzSIBA, $S$-isobutyl-1-deazaadenosine; 3-dzSIBA, $S$-isobutyl-3-deazaadenosine; 7-dzSIBA; $S$-isobutyl-7-deazaadenosine.
} 
Ethylene, the simplest among the well-characterized plant hormones, occurs in the gas state under normal physiological conditions. Its production ranges from almost 0 up to $500 \mathrm{nl} / \mathrm{g}$ of plant tissue per h but its biological activity is observed even when its concentration is very low (as little as $10-100 \mathrm{nl} / \mathrm{l}$ of air) (Theologis, 1992). For example, ethylene effects include promotion of fruit ripening, flower senescence, petal and leaf abscission, inhibition of seedling elongation, and stimulation of root initiation (Lincoln et al., 1993; Abel et al., 1995; Clark et al., 1997; Kamachi et al., 1997; Trebish et al., 1997; Nakatsuka et al., 1998; Barry et al., 2000; Ishiki et al., 2000; Llop-Tous et al., 2000). Ethylene production in plant tissues in response to biotic and abiotic stress (wounding, cold, anaerobiosis, $\mathrm{Cd}$, ozon, pathogens, etc.) is a signalling mechanism of profound physiological consequences (Spanu et al., 1993; Avni et al., 1994; Olson et al., 1995; Lelievre et al., 1997; Schlagnhaufer et al., 1997; Tuomainen et al., 1997; Zarembinski \& Theologis, 1997; Kathiseran et al., 1998; Arteca \& Arteca, 1999; Tatsuki \& Mori, 1999; Kato et al., 2000).

The ethylene biosynthetic pathway of higher plants involves the following steps:

1) Methionine adenosyltransferase (ATP:methionine $S$-adenosyltransferase, EC 2.5.1.6) catalyzes the conversion of methionine into $S$-adenosylmethionine (SAM), reaction 1;

2) 1-Aminocyclopropane-1-carboxylate synthase ( $S$-adenosyl-L-methionine methylthioadenosine lyase, EC 4.4.1.14) catalyzes the synthesis of 1-aminocyclopropane-1-carboxylic acid (ACC) from SAM, reaction 2;

3) ACC is converted to ethylene by ACC oxidase, reaction 3 (Yang \& Hoffman, 1984; Kende, 1993; Zarembinski \& Theologis, 1994).

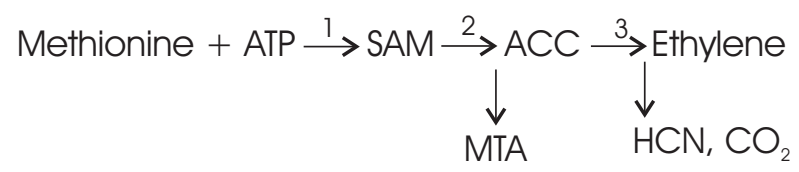

ACC synthase is the key regulatory enzyme in ethylene synthesis in higher plants (step 2).
The regulatory effect of steps 1 and 3 is not so important because SAM is constantly synthesized and also utilized by other reactions, such as methylation and polyamine synthesis, whereas ACC oxidase activity is most often constitutive (Botha \& Whitehead, 1992; Li et $a l .$, 1992).

Besides ACC, ACC synthase produces 5'-methylthioadenosine (MTA). MTA is recycled into methionine by the Yang cycle (Yang \& Hofman, 1984; Zarembinski \& Theologis, 1994). This cycle allows high rates of ethylene production without high intracellular concentrations of methionine. It involves the following steps:

5 '-methylthioadenosine (MTA) $\rightarrow$

5 '-methylthioribose (MTR) $\rightarrow$

5 '-methylthioribose-1-phosphate (MTR-1-P) $\rightarrow$ 2-keto-4-methylthiobutyrate (KMB) $\rightarrow$ methionine

ACC may be also converted to 1-(malonylamino)cyclopropane-1-carboxylic acid (MACC) by ACC $N$-malonyltransferase. Malonylation of ACC can decrease free ACC level in plant tissues and is irreversible under physiological conditions. $\mathrm{HCN}$ is one of the products during the conversion of ACC to ethylene but higher plants possess an effective system of its detoxification (Yip \& Yang, 1988).

Ethylene controls its own synthesis and in higher plants two systems of ethylene production operate. System 1 operates in all vegetative tissues and in the fruit before its ripening, system 2 during the ripening of climacteric fruit and during petal senescence. Ethylene shows auto-inhibitory effect in system 1 but auto-stimulatory effect in system 2 (Barry et $a l ., 2000)$.

Because of large loss of fruit, flowers and vegetables due to ethylene's effects on ripening and senescence, attempts have been made to prevent or delay fruit ripening in a reversible manner. ACC synthase activity has been demonstrated by antisense RNA experiments in to- 
matoes as the rate-limiting step in ethylene synthesis. Transgenic tomatoes expressing ACC synthase antisense RNA do not ripen unless exposed to exogenous ethylene (Rottmann et al., 1991; Theologis et al., 1993). Transgenic tomatoes with introduced ACC deaminase gene are characterized by a reduced ACC level which results in delayed fruit ripening (Klee et al., 1991; Theologis et al., 1992).

Microorganisms liberate ethylene as a product of metabolism of: a) 2-oxoglutarate, b) 2-oxo-4-methylthiobutyrate. These pathways do not involve ACC as the intermediate. The fungus Penicillium citrinum is the first reported microorganism which is able to synthesize ACC from SAM and to degrade it into ammonia and 2-oxobutyrate by the action of ACC deaminase (Jia et al., 1999; Kakuta et al., 2001).

\section{REACTION MECHANISM OF THE CONVERSION OF SAM TO ACC AND MTA CATALYZED BY ACC SYNTHASE}

ACC synthase belongs to the family of pyridoxal 5'-phosphate-dependent enzymes. In PLP-dependent enzymes, the formyl group at position 4 of pyridoxal phosphate forms an aldimine bond with the $\varepsilon$-amino group of a specific lysine residue, forming a Schiffs base within the active site of the enzyme. ACC synthases have absorption maxima between 426 and $431 \mathrm{~nm}$, which is probably due to the internal Schiff base (Satoh et al., 1993).

A proposed reaction mechanism - the conversion of SAM to ACC by ACC synthase - involves the following steps:

1. The active site lysine of ACC synthase forms a Schiff base (internal aldimine) with the bound PLP in unligated enzyme (Fig. 1a).

2 . The amino group of the substrate SAM replaces that of active site lysine as the Schiff base partner of the cofactor (external aldimine), (Fig. 1a).

3 . The C- $\alpha$ proton of SAM is abstracted by the $\varepsilon-\mathrm{NH}_{2}$ function of active site lysine to form a resonance-stabilized carbanion (Fig. 1a), that in turn attacs the C- $\gamma$ atom (Fig. 1b) to expel 5'-methylthioadenosine (MTA), with the formation of the imine of ACC (Fig. 1b, 1c).

4. The product is released upon transimination by active site lysine to complete the catalytic sequence (Fig. 1d).

The scheme of ethylene synthesis with the atoms of methionine which are the precursors of ethylene, marked by bold type set, is presented in Fig. 1e.

\section{THE MECHANISM-BASED \\ INACTIVATION OF ACC SYNTHASE \\ BY THE SUBSTRATE SAM, AND OTHER ACTIVITIES OF THIS ENZYME}

ACS is inactivated by its substrate SAM in an irreversible manner (mechanism-based inactivation). ACC synthase is optimized to direct electrons from the quinonoid intermediate to the $\gamma$-carbon of its substrate-SAM (Fig. 1b), to yield ACC and MTA. SAM exists as an $S, S$ or $R, S$ diastereomeric mixture (isomers with respect to its sulfonium centre); only the $S, S$ diastereomer yields ACC. The $R, S$ form inactivates the enzyme. The inactivation of ACC synthase is a result of a nucleophilic attack on residual enzyme-bound L-vinylglycine (L-VG) originating from the $\beta, \gamma$-elimination of MTA from the internal SAM aldimine (Satoh \& Esashi, 1986; Casas et al., 1993; White et al., 1994; Feng \& Kirsch, 2000). A scheme of the proposed mechanism-based inactivation of ACS is shown in Fig. 2.

In vivo SAM leads to the formation of L-vinylglycine, causing alkylation and inactivation of the enzyme about once in 30000 turnovers (Oetiker et al., 1997). The peptide Ser-Leu-Ser-X-Asp-Leu-Gly-Leu-Pro-Gly-Phe-Arg from SAM-inactivated ACC synthase from apple was isolated. $\mathrm{X}$ was a modified amino acid (alkylation product) coincident with the position of active-site Lys273 (Satoh et al., 1993; Feng \& Kirsch, 2000). 

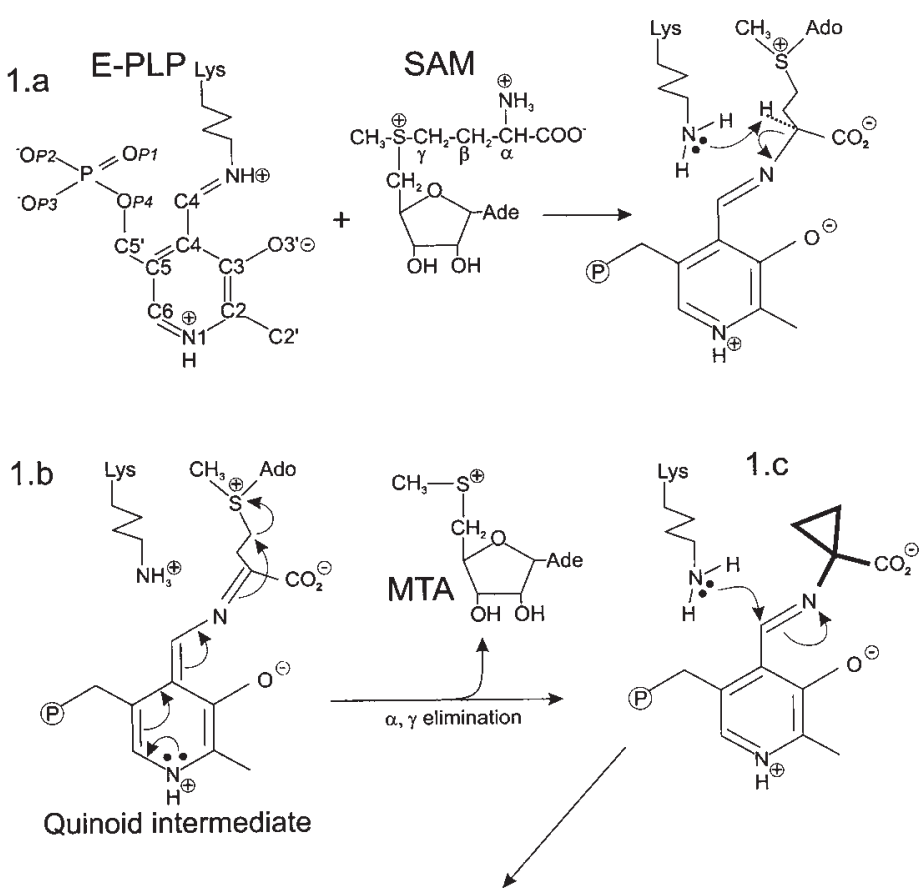

$1 . d$<smiles></smiles>

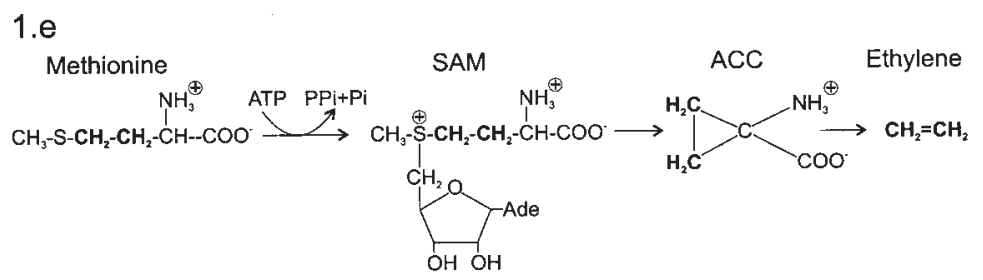

Figure 1. Scheme of reaction catalyzed by 1-aminocyclopropane-1-carboxylate synthase.

Denotations: OP1-OP4, oxygens of the pyridoxal 5'-phosphate moiety. Details in text.

The half-life of ACS was found to be 25 min in mung bean hypocotyl segments treated with auxin but 30-40 minutes in wounded green tomato pericarp, and $114 \mathrm{~min}$ in ripening tomato pericarp. The mechanism-based inactivation of ACC synthase plays a pivotal role in the rapid decrease in the enzymatic activity that was observed in plant tissue (Satoh \& Esashi, 1986; Satoh et al., 1993).

ACC synthase reveals some additional catalytic activity apart from the preferred physiological role. L-Vinylglycine is an alternative substrate as well as a mechanism-based inhibitor of ACS. The high molar ratio of L-VG/ACS (over 5000) is necessary for a complete inactivation of ACS.
L-vinylglycine + ACS-PLP $\rightarrow$

vinylglycine-PLP-ACS $\rightarrow$

alkylated inactive ACS + PLP

The majority of L-VG (98.2\%) is converted to a product other than the inactive enzyme. The primary reaction is the conversion of L-VG to $\alpha$-ketobutyrate and ammonia:

$$
\begin{aligned}
& \mathrm{CH}_{2}=\mathrm{CH}-\mathrm{CH}\left(\mathrm{NH}_{3}^{+}\right)-\mathrm{COO}^{-} \rightarrow \\
& \mathrm{CH}_{3}-\mathrm{CH}_{2}-\mathrm{CO}_{-} \mathrm{COO}^{-}+\mathrm{NH}_{4}^{+}
\end{aligned}
$$

The L-VG deaminase activity of ACC synthase yields the products $\alpha$-ketobutyrate 


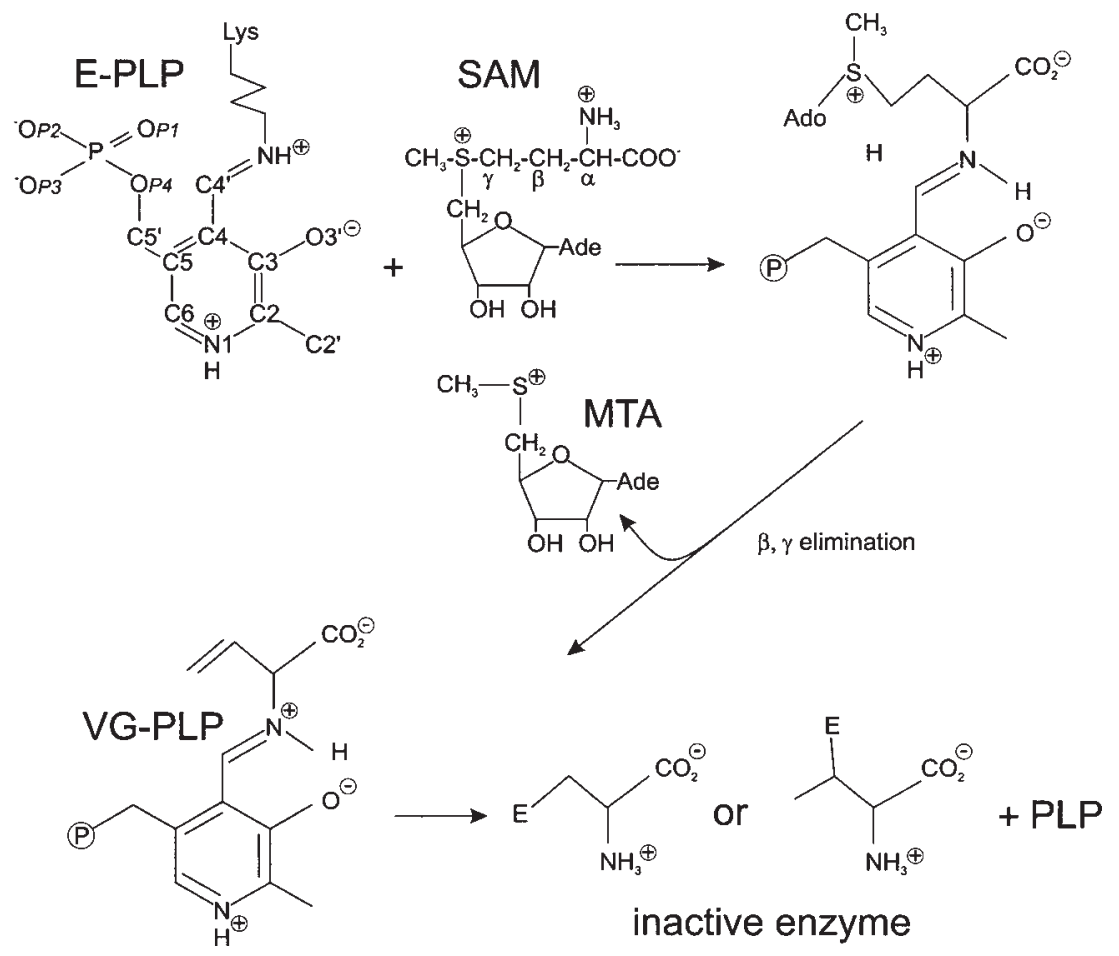

Figure 2. Model of mechanism-based inactivation of 1-aminocyclopropane-1-carboxylate synthase by its substrate SAM.

Denotations: OP1-OP4, oxygens of the pyridoxal 5'-phosphate moiety; VG-PLP, vinylglycine-PLP. Details in text.

and ammonia with a $k_{\text {cat }}$ value of $1.8 \mathrm{~s}^{-1}$ and a $K_{\mathrm{m}}$ value of $1.4 \mathrm{mM}$ (Feng \& Kirsch, 2000).

Feng et al. (2000) investigated the aminotransferase activity of ACC synthase from apple and characterized it as non- $\alpha$-ketoglutaratecoupled. PLP is reductively aminated to PMP (pyridoxalamine) by transamination of an amino acid to its corresponding $\alpha$-keto acid:

amino acid + ACS-PLP $\rightarrow$

keto acid + ACS-PMP

The enzyme exhibits substrate specifity in transamination with the preference of L-Ala $>$ L-Arg > L-Phe > L-Asp. Transamination of L-Asp and L-Phe by ACC synthase is very slow. Addition of excess pyruvate converts the PMP form of the enzyme back to the PLP form. The PMP form of ACC synthase is quite unstable. This unstability would generate apoenzyme which would capture PLP to restore the physiologically active form of ACS.

\section{INHIBITORS OF ACC SYNTHASE ACTIVITY}

ACC synthase activity may be reversibly regulated by various substances associated with the methionine-recycling pathway, SAM metabolism, polyamine synthesis and natural and chemical analogs of SAM or inhibitors of pyridoxal phosphate-linked enzymes.

MTA and KMB, intermediates of the methionine-recycling pathway inhibit the enzyme activity in an uncompetitive and noncompetitive fashion, respectively (Hyodo \& Tanaka, 1986).

Sinefungin (a naturally occuring antifungal antibiotic isolated from Streptomyces griseus), SAHC, 3dz-SAH, SIBA, 1dz-SIBA, 3dz-SIBA, 7dz-SIBA are analogs to SAM (Fig. 3). When tested with ACC synthase from tomatoes the order of decreasing inhibitory activity of the SAM analogs was as follows: sinefungin > SAHC and SAHC derivates $>S$-n-butyladenosine and SIBA. The inhibitors tested can be divided into two groups, those modified in the adenine moiety and those modified in the $5^{\prime}$ 
Table 1. Comparison of amino acids number (aa), molecular masses (kDa) and isoelectric points (pI) of some well-characterized ACS isoenzymes from different plants

\begin{tabular}{|c|c|c|c|c|}
\hline ACS isoenzyme & aa number & $\begin{array}{l}\text { Molecular } \\
\text { mass (kDa) }\end{array}$ & $\mathrm{pI}$ & References \\
\hline \multicolumn{5}{|c|}{ Lycopersicon esculentum } \\
\hline LE-ACS1A & 485 & 54.8 & 5.94 & (Rottmann et al., 1991) \\
\hline LE-ACS1B & 483 & 54.6 & 6.16 & (Rottmann et al., 1991) \\
\hline LE-ACS2 & 485 & 54.7 & 7.71 & (Rottmann et al., 1991) \\
\hline LE-ACS3 & 469 & 53.1 & 8.01 & (Rottmann et al., 1991) \\
\hline LE-ACS4 & 476 & 53.5 & 5.40 & (Rottmann et al., 1991) \\
\hline LE-ACS7 & 467 & 53.1 & 8.30 & (Shiu et al., 1998) \\
\hline \multicolumn{5}{|c|}{ Solanum tuberosum } \\
\hline ST-ACS1A & 465 & 52.9 & 7.86 & (Destefano-Beltran et al., 1995) \\
\hline ST-ACS1B & 465 & 52.9 & 8.23 & (Destefano-Beltran et al., 1995) \\
\hline ST-ACS2 & 441 & 50.2 & 5.74 & (Destefano-Beltran, 1995) \\
\hline \multicolumn{5}{|l|}{ Lupinus albus } \\
\hline LA-ACS1 & 487 & 55.0 & 7.06 & (Bekman et al., 2000) \\
\hline LA-ACS3 & 488 & 55.0 & 7.61 & (Bekman et al., 2000) \\
\hline LA-ACS4 & 445 & 51.0 & 6.58 & (Bekman et al., 2000) \\
\hline LA-ACS5 & 448 & 51.0 & 6.44 & (Bekman et al., 2000) \\
\hline \multicolumn{5}{|l|}{ Vigna radiata } \\
\hline VR-ACS1 & 484 & 54.6 & 6.0 & (Botella et al., 1992) \\
\hline \multicolumn{5}{|c|}{ Arabidopsis thaliana } \\
\hline AT-ACS1 & 496 & 54.6 & 7.3 & (Van Der Straeten et al., 1992) \\
\hline AT-ACS2 & 496 & 55.6 & 7.3 & (Liang et al., 1992) \\
\hline AT-ACS4 & 474 & 53.8 & $8.2,8.4$ & (Abel et al., 1995; Liang et al., 1995) \\
\hline AT-ACS5 & 470 & 53.3 & 7.5 & (Liang et al., 1995) \\
\hline \multicolumn{5}{|l|}{ Brassica oleraceae } \\
\hline BO-ACS2 & 491 & 55.0 & 5.9 & (Pogson et al., 1995) \\
\hline \multicolumn{5}{|l|}{ Cucurbita pepo } \\
\hline CP-ACS1A & 493 & 55.8 & 7.07 & (Huang et al., 1991) \\
\hline CP-ACS1B & 494 & 55.9 & 7.68 & (Huang et al., 1991) \\
\hline \multicolumn{5}{|l|}{ Cucurbita maxima } \\
\hline CM-ACS & 493 & 55.8 & 5.0 & (Sato et al., 1991) \\
\hline \multicolumn{5}{|l|}{ Cucumis melo } \\
\hline CMe-ACS1 & 493 & 56.0 & 6.41 & (Miki et al., 1995) \\
\hline \multicolumn{5}{|l|}{ Citrus chinensis } \\
\hline CS-ACS1 & 483 & 54.1 & 6.63 & (Wong et al., 1999) \\
\hline CS-ACS2 & 477 & 53.3 & 6.72 & (Wong et al., 1999) \\
\hline \multicolumn{5}{|c|}{ Pelargonium hortorum } \\
\hline G-ACS1 & 482 & 54.1 & 6.4 & (Wang \& Arteca, 1995) \\
\hline \multicolumn{5}{|l|}{ Oryza sativa } \\
\hline OS-ACS1 & 487 & 53.1 & 8.2 & (Zarembinski \& Theologis, 1993) \\
\hline
\end{tabular}

substituent. The 7-nitrogen of adenine is apparently necessary for the inhibitory activity, judging from the inactivity of $7 \mathrm{dz}$-SIBA. Although 3dz-SIBA was inactive, the inhibitory activity of 3dz-SAHC suggests that the $5^{\prime}$ substituent containing an amino group may be important for inhibition (Icekson \& Apelbaum, 1983; Miura \& Chiang, 1985).

SAM serves as an important common precursor for the pathway of polyamine and ethylene synthesis. The interactions between ethylene and polyamine metabolism are discussed and 
many conflicting reports have been published on the effect of exogenously applied polyamines on ethylene synthesis. At physiological $\mathrm{pH}$, polyamines are fully protonated and polycationic. It is possible that the amino group of amines prevent SAM from reacting with ACC synthase by interfering with the prostetic groups of the enzyme. Polyamines such as putrescine, spermidine and spermine inhibit ACC synthase activity in a noncompetitive manner. It has been suggested that polyamines also suppress the induction of the enzyme. From the physiological point of view, it is of interest to note that while ethylene is a plant-aging hormone leading to retardation of growth and promotion of senescence, polyamines delay senescence in some plant species (Hyodo \& Tanaka, 1986; Botha \& Whitehead, 1992).

The hydroxylamine or vinylglycine analogs are potent inhibitors of PLP-dependent enzymes activity in vivo and in vitro. The hydroxylamine analogs inactivate the enzyme forming stable oximes with PLP.

Several vinylglycine analogs are shown below.

$\begin{array}{ll}\text { Rhizobitoxine } & \begin{array}{l}\mathrm{HOCH}{ }_{2}-\mathrm{CH}\left(\mathrm{NH}_{2}\right)-\mathrm{O}-\mathrm{CH}=\mathrm{CH}-\mathrm{CH} \\ \left(\mathrm{NH}_{2}\right)-\mathrm{COOH}\end{array} \\ & \mathrm{NH}_{2}-\mathrm{CH}_{2}-\mathrm{CH}_{2}-\mathrm{O}-\mathrm{CH}=\mathrm{CH}-\mathrm{CH} \\ & \left(\mathrm{NH}_{2}\right)-\mathrm{COOH}\end{array}$

MVG $\quad \mathrm{CH}_{3}-\mathrm{O}-\mathrm{CH}=\mathrm{CH}-\mathrm{CH}\left(\mathrm{NH}_{2}\right)-\mathrm{COOH}$

AVG has the strongest inhibitory effect on ACC synthase among the vinylglycine analogs tested (Yang \& Hoffman, 1984). Rhizobitoxine [2-amino-4-(2-amino-3-hydropropoxy)trans-but-3-enoic acid] is synthesized by the legume symbiont Bradyrhizobium elkanii and the broad-host-range plant pathogen Burkholderia andropogonis (Yasuta et al., 1999). The bacterial toxin induces foliar chlorosis of the host plant but the role of the toxin in symbiosis and pathogenicity has not been resolved. It was first isolated from root nodules produced by Rhizobium japonicum in soybean. Rhizobitoxine inhibits $\beta$-cystathionase and
ACC synthase in the methionine and ethylene biosynthetic pathway, respectively.

Aminoethoxyvinylglycine (AVG) was isolated from a Streptomyces strain, the methoxy analog (MVG) was discovered in fermentation broth of Pseudomonas aeruginosa (Mattoo et al., 1979). These unsaturated enol ether amino acids are potent inhibitors of ethylene synthesis in higher plants. It is interesting that both symbiotic and pathogenic bacteria produce ACC synthase inhibitors structurally and enzymologically similar to rhizobitoxine so as to control the ethylene-induced plant response that would prevent a successful infection.

The crystal structures of tomato ACS in complex with AVG and PLP imply that the competitive inhibitor AVG binds close to PLP. It is a good position for the formation the external aldimine with PLP. The $\alpha$-amino group of AVG is located $4 \AA$ away from $\mathrm{C}^{\prime}$ ' of the pyridine ring of PLP. The $\alpha$-carboxylate group of AVG forms the three following hydrogen bonds - with the backbone nitrogen of alanine, the guanidine nitrogen of arginine (in tomato ACS Ala54, Arg412) and water. The amine group at the other end of AVG forms a van der Waals contact with a specific tyrosine residue (in tomato ACS Tyr 152). Probably the $\alpha$-amino and $\alpha$-carboxylate groups of AVG simulate SAM binding in ACS (Huai et al., 2001).

\section{THE STRUCTURE OF ACC SYNTHASE AND AMINO-ACID RESIDUES CRITICAL FOR ITS FUNCTION}

ACC synthase, an enzyme located in the cytoplasm, is encoded by a highly divergent multigene family but the low content of ACS polypeptides $(0.0001 \%-0.001 \%$ of plant soluble proteins) has strongly limited characterisation of these proteins. The expression of functional ACS cDNAs in heterologous systems provides an opportunity to explore these enzymes. The first ACS cDNA was cloned 


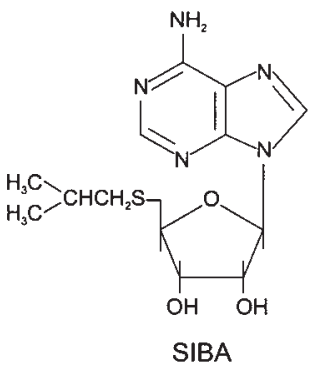

(S-Isobutyladenosine)

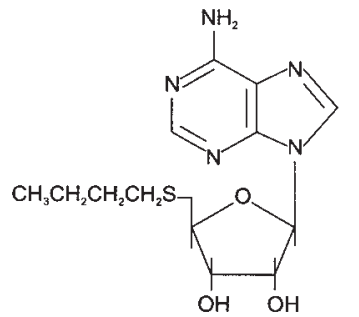

S-n-Butyladenosine

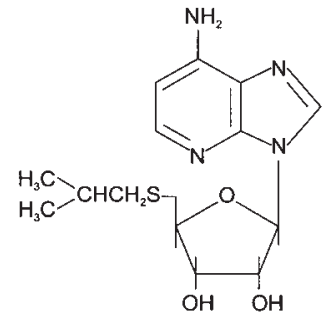

1-dz SIBA

(S-Isobutyl-1-deazaadenosine)
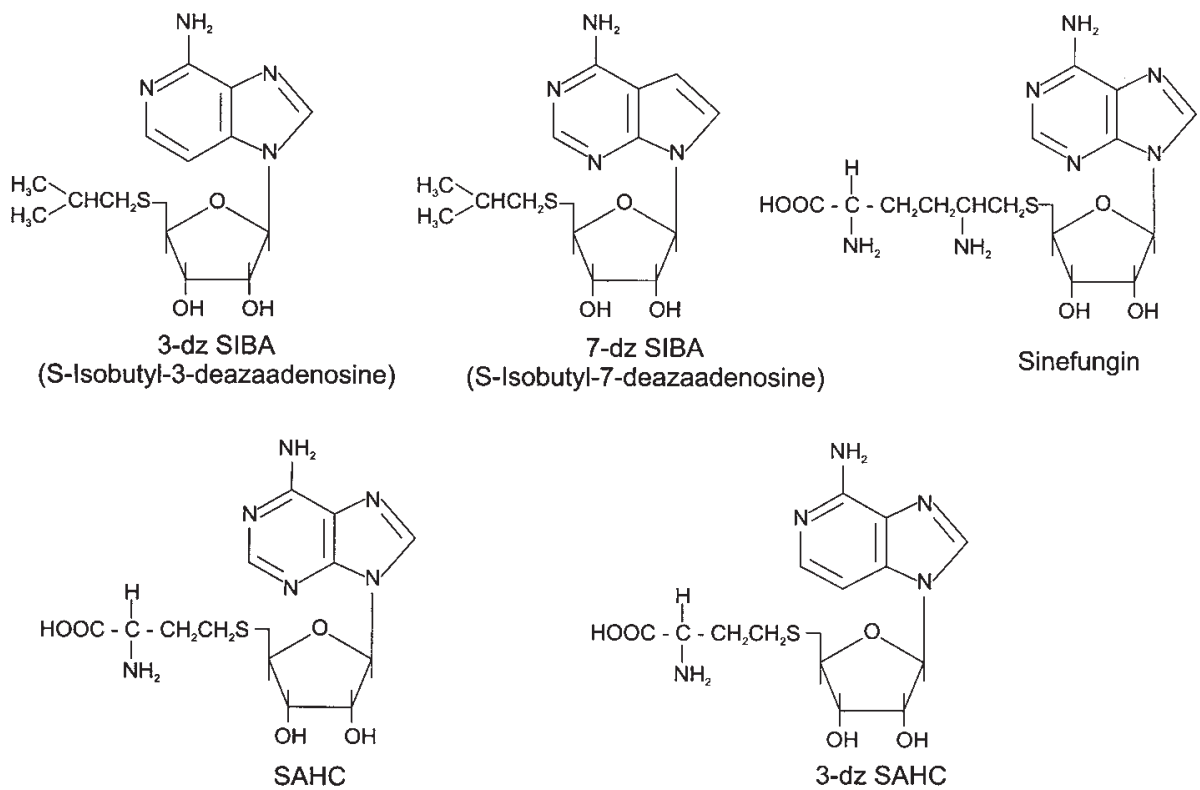

(S-Adenosylhomocysteine)

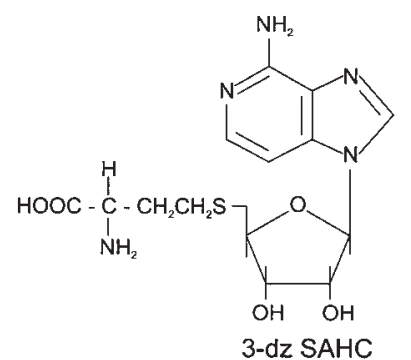

(3-Deazaadenosylhomocysteine)

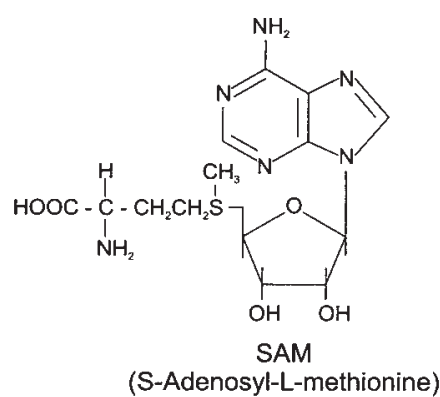

Figure 3. Natural and synthetic analogs of SAM.

from zucchini fruit tissue and overexpressed in E. coli and yeast (Zarembinski \& Theologis, 1994; Abel et al., 1995).

Despite the polymorphic character of the multigene family of ACS isoenzymes their primary structures show similar molecular size (441aa to 496aa) and seven strongly conserved regions (Wong et al., 1999). ACC synthase from carnation is the only exception characterised to date. It is larger than others (516aa) because of the presence of a stretch of
18 threonine residues (ACA) at position 452-469aa encoded by repeats of the CAA sequence. The stretch of (CAA) 18 probably represents a highly repeated element within the carnation genome and is not unique to the ACC synthase gene (Park et al., 1992).

A necessary feature of the polypeptide during its attachment to the ACS family is the PLP-binding site (region 5): -Ser-Leu-Ser-LysAsp-Leu/Met-Gly-Leu-Pro-Gly-Phe-Arg-. The highly conserved regions (regions 1 to 7 ) 
among all plant ACS are shown below (the position of residues numbered according to the 485aa long polypeptide of LE-ACS2 from tomato).

\begin{tabular}{c|l}
1 & -49 Ile-Gln-Met-Gly-Leu-Ala-Glu-Asn-Gln-Leu 58- \\
\hline 2 & -89 Phe-Gln-Asp-Tyr-His-Gly-Leu-Pro 97- \\
\hline 3 & -136 Phe-Cys-Leu-Ala-Asp-Pro-Gly 142- \\
\hline 4 & -205 Thr-Asn-Pro-Ser-Asn-Pro-Leu-Gly-Thr 213- \\
\hline 5 & -274 Tyr-Ser-Leu-Ser-Lys-Asp-Met-Gly- \\
& Leu-Pro-Gly-Phe-Arg-Val-Gly 288- \\
\hline 6 & -304 Met-Ser-Ser-Phe-Gly-Leu-Val-Ser 311- \\
\hline 7 & -408 Pro-Gly-Trp-Phe-Arg-Val-Cys-Phe-Ala-Gln 417-
\end{tabular}

The roles of amino-acid residues critical for ACC synthase activity have been elucidated by site-directed or random mutagenesis investigations performed on ACC synthases from apple and tomato (White et al., 1994; Li et al., 1997; Tarun et al., 1998; Capitani et al., 1999; Zhou et al., 1999; Huai et al., 2001; McCarthy et al., 2001). The residues important for catalysis and structure of ACC synthase from tomato are shown in Fig. 4 and are listed below (the numbers in brackets indicate equivalent residue in ACS from apple according to Capitani et al., 1999).

Lys278 (273): active site lysine forming the Schiff base with the bound PLP in the unligated enzyme. Mutated enzymes from apple and tomato with the active-site lysine replaced by alanine have no detectable activity.

Asn209 (202), Tyr240 (233): 03 of the pyridine ring of PLP forms two hydrogen bonds with these amino-acid residues, Tyr240 attracts the proton of Lys278 to promote the formation of the external aldimine.

Tyr152 (145): stabilization of the pyridine ring of PLP and catalytic function. Tyr 152 performs a nucleophilic attack to break the C- $\gamma-\mathrm{S}$ bond what leads to the formation of the key intermediate - ACC-aldimine (the $\mathrm{OH}$ group of Tyr152 is positioned 3.7 and $3.5 \AA$ from $\mathrm{C}-\gamma$ and $\mathrm{S}$ of SAM, respectively).

Asp237 (230): hydrogen bond with N1 of PLP.

Ala127 (120), Thr128 (121), Ser275 (270), Ser277 (272), Arg286 (281): residues required for correct orientation of PLP in the active site during formation of external aldimine between SAM and PLP, hydrogen bonds with the phosphate oxygens of PLP.

Glu55 (47): putative ionic interaction between SAM and this residue positioned near the sulfonium pole of the $(S, S)$-diastereomer of SAM.

Ala54 (46), Arg412 (407): $\alpha$-carboxylate group of SAM forms hydrogen bonds with the nitrogen of alanine and the guanidino moiety of arginine.

Arg157 (150): $\mathrm{O}^{\prime}$ and $\mathrm{O}^{\prime}$ atoms of the sugar of SAM form hydrogen bonds with the guanidine group of arginine.

Pro26 (Ser18), Tyr27 (19), Phe28 (20), Pro153 (144): these residues contribute in a hydrophobic pocket for the adenine ring of SAM.

Tyr92 (85): the complete conservation of this tyrosine residue among all known ACS suggests that it may be involved in substrate recognition. In the apple, Tyr85 to Ala85 mutation leads to an inactive ACC synthase, whereas in the tomato Tyr92 to Phe92 or Trp92 mutations produce a partially active enzyme, while substitutions Tyr92 to His92 or Leu92 inactivate the enzyme. In contrast, according to Tarun et al. (1998), Tyr92 residue from tomato ACS is not essential for the activity of the enzyme but it may play a role in binding the PLP cofactor and in making intersubunit contacts in the dimeric form of ACS (Tarun et al., 1998; Capitani et al., 1999).

Replacement of Tyr233 with Phe233 in ACS from apple caused a 24-fold increase in $K_{\mathrm{m}}$ for SAM (White et al., 1994). This tyrosine residue is not absolutely conserved in all ACS one of the ACC synthases from carnation shows a Phe residue at this position (Tarun et al., 1998).

Substitution of Arg286 with Leu286 in tomato ACS results in the mutant's lower affinity for both PLP and SAM. The loss of the correct orientation of PLP at the active site of the mutant with Leu286 decreases the binding affinity of the enzyme for SAM. Moreover, the 
Leu286 mutant shows the reduction in $k_{\text {cat }}$ which suggests that Arg286 participates in the substrate catalysis. The replacement of Arg286 with Val, Thr, Ile, and Ala converts tomato ACS into mutants losing $95 \%$ to $100 \%$ of the original catalytic activity (Tarun et al., 1998).

The mutation of Arg407 (tomato Arg412) to Lys407 in ACS from apple results in at least 20-fold increase in $K_{\mathrm{m}}$ for SAM (White et al., 1994).

The N-termini of ACS are characterised by two highly conserved leucine and serine residues (Leu12 and Ser13 in tomato ACS, Leu4 and Ser5 in apple ACS). The removal of ten of proteins of very similar molecular masses which differ from each other in the length of the C-truncated fragments. These values for winter squash and tomato ACS are lower by 6-8 kDa than those calculated from the corresponding cDNA. Purified wound-inducible tomato ACC synthase appears as two distinct processed polypeptides, despite the presence of protease inhibitors in the purification procedure. This suggests that the nature of the C-terminus of ACC synthase makes it susceptible to cleavage. The processing seems to occur invariably when the tissue is wounded or when cells are homogenized. The monomeric deletion mutant (tomato ACS) of the last 52aa

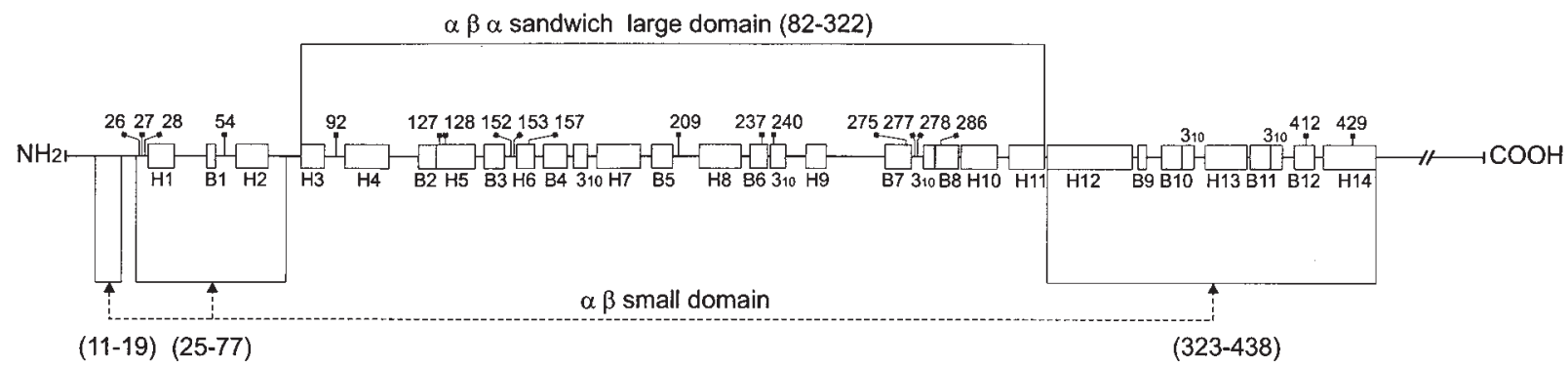

Figure 4. Schematic presentation of tomato ACS polypeptide with marked $\alpha$-helical and $\beta$-stranded secondary structure regions, residues critical for catalysis and fragments of the polypeptide representing the large and the small domain in the three-dimensional structure of enzyme.

Denotations: open blocks, $\alpha$-helical regions (H1-H14), filled blocks, $\beta$-strand (B1-B12) and helix $3_{10}$ regions.

residues from the $\mathrm{N}$-terminus of tomato ACS leads to a slight increase in ACC synthase activity but a deletion of any more residues would result in a substantial reduction in the activity. Addition of several amino-acid residues to the N-terminus does not affect the formation of the dimeric protein (Satoh et al., 1993; Li et al., 1996; Tarun et al., 1998).

The carboxyl termini of all known ACC synthases are quite divergent and consist of a hypervariable region of 18-85 C-terminal residues (Wong et al., 1999). Mutations in this hypervariable region (for tomato LE-ACS2 in the region of the last 56 residues) do not lead to any decrease in the enzyme activity.

ACS polypeptides prepared from plant tissues or overexpression systems are mixtures
C-terminal residues shows a dramatic increase in activity. Further deletion of the C-terminus through Arg429 results in a complete inactivation of the enzyme. Limited C-terminal proteolysis of ACC synthase may occur naturally and certain endogenous or exogenous stimuli cause the activation of ACC synthase by forming the enzymes with the higher specific activity. The phenomenon may be attributed to the release of proteases upon wounding and during tissue senescence (Kende, 1993; Satoh et al., 1993; Li \& Mattoo, 1994; Zarembinski \& Theologis, 1994; Tarun et al., 1998).

The subunit structure of ACS is controversial (Nakajima et al., 1988; Rottmann et al., 1991; Lincoln et al., 1993; Oetiker et al., 1997; 
Huxtable et al., 1998). ACS enzymes from mung bean hypocotyls and a wound-inducible ACC synthase from zucchini have dimeric forms. Satoh et al. (1993) report that enzymes from ripe tomato are monomers, whereas the same enzymes overexpressed from tomato ACS cDNAs occur in dimeric forms. In contrast, Li \& Mattoo (1995) report that tomato LE-ACS2 isoenzyme from fruit is a dimer whereas its carboxyl terminal deletion mutants (with last 46 and 52 residues deleted) function as highly active monomers. According to Tarun \& Theologis (1998) the highly active carboxyl terminal deletion mutant LE-ACS2 with last 46 residues deleted is able to dimerize.

The catalytically inactive tomato and apple ACS mutants with substitutions of the highly conserved tyrosine or lysine residues and the hyperactive mutant with 46 C-terminal amino-acid residues deleted were coexpressed in E. coli (Li et al., 1997; Tarun \& Theologis, 1998). The catalytic activity of ACS was measured. The conclusions are the following:

1) an active dimer may be formed from two different properly folded defective monomer subunits.

2) the hyperactive ACS monomer is able to dimerize with inactive monomer, which results in a reduced activity.

3) two inactive monomers of different tomato isoenzymes are able to undergo heterodimerization and show limited restoration of activity.

The question is whether ACS may be active both as a monomer and as a dimer. On the basis of the experimental data, we cannot distinguish these two possibilities.

The AT-ACS1 polypeptide from Arabidopsis thaliana is missing the highly conserved tripeptide Thr-Asn-Pro (region 4) and has no ACS activity. It is possible that AT-ACS1 may act as a dominant negative regulator of other ACS by forming heterodimers (Liang et al., 1995).

Satoh et al. (1993) suggest that the dimeric forms of tomato ACS isoenzymes (LE-ACS2 and LE-ACS4) are more sensitive to mechanism-based inactivation than their monomers because of minor differences in the parameters of the active sites of these two forms. The concentration of SAM localized in the protoplasm in ripe tomato pericarp (higher than 100 micromolar) should almost saturate the enzyme's active site. Changes in the sensitivity of the enzyme to mechanism-based inactivation probably take part in the regulation of ethylene synthesis in plant tissue.

Despite the quite similar molecular masses of ACS isoenzymes their isoelectric points differ considerably, as shown in Table 1.

Penicillium citrinum synthesizes ACC from SAM and metabolizes ACC to ammonia and 2-oxobutyrate, not to ethylene (step 3, ACC deaminase).

$$
\begin{aligned}
& \text { Methionine }+ \text { ATP } \stackrel{1}{\longrightarrow} \mathrm{SAM}^{2} \stackrel{2}{\longrightarrow} \\
& \mathrm{ACC} \stackrel{3}{\longrightarrow} \text { 2-oxobutyrate }+ \text { ammonia }
\end{aligned}
$$

The ACC synthase from Penicillium citrinum is a 430aa long polypeptide (a shorter C-terminus than that in plant enzymes) with molecular mass $48 \mathrm{kDa}$. The native form of the enzyme is a dimer. ACS from $P$. citrinum has $K_{\mathrm{m}}$ for SAM about 100-fold higher than plant ACS (Jia et al., 1999). Five of the seven highly conserved regions (regions 1, 3, 4, 5, 7) found in plant ACS occur in ACS from $P$. citrinum and have $45-50 \%$ of residues identical with their plant counterparts. $P$. citrinum ACS contains six highly conserved residues responsible for the binding of PLP and SAM (Lys268, Tyr225, Asn194, Asp222, Arg276, Arg408) and Arg424 corresponding to the conserved Arg429 of the tomato enzyme. The highly conserved tyrosine residue - Tyr85 or Tyr92 (in apple or tomato ACS) is not conserved and Gly74 is at the equivalent position in P. citrinum ACS (Kakuta et al., 2001).

An artificial bifuncional enzyme - ACSO (90 $\mathrm{kDa}$ ) was created by in-frame fusion of a C-terminus truncated ACC synthase from soybean and an N-terminus truncated ACC oxidase from tomato. ACSO was able to con- 
vert SAM to ethylene. Native ACSO is oligomeric, about $360 \mathrm{kDa}$, suggesting that this fusion enzyme has a tetrameric form ( $\mathrm{Li}$ et al. 1996).

\section{THE CRYSTAL STRUCTURE OF ACC SYNTHASE}

The crystal structure of ACC synthase from apple without the PLP cofactor and two crystal structures of tomato ACS: with PLP and with PLP and the inhibitor AVG were described (Hohenester et al., 1994; Capitani et al., 1999; Huai et al., 2001). The structures with and without PLP represent dimeric forms but the structure that with PLP and AVG is a monomer. The residues involved in catalysis and PLP and AVG interaction are located within the active site of the monomer. The monomeric form of tomato ACS implies should be sufficient to catalysis.

A schematic presentation of the secondary structures building the large and the small domain in the three-dimensional structure of ACS from tomato is shown in Fig. 4. The large domain $(\alpha / \beta / \alpha$ sandwich domain) contains a central several strand $\beta$-sheet flanked by nine $\alpha$-helices (residues $82-322$ ). The small domain ( $\alpha / \beta$ domain) comprises a five $\beta$ strands and five $\alpha$-helices (residues 11-19, 25-77, 323-438), (Huai et al., 2001).

The full-length polypeptide from apple (52 $\mathrm{kDa}$ ) is C-terminally truncated to a shorter form $(48 \mathrm{kDa})$ by unknown proteolytic processes upon storage. Only the truncated form has been crystallized to date. ACC synthase from apple is a two-domain protein with each domain composed of a central sheet of $\beta$-strands connected by $\alpha$-helices packed on both sides. The large domain of this ACC synthase contains a seven-stranded $\beta$-sheet, the central part of the small domain consists of a four-stranded antiparallel and a twostranded parallel sheet. In the dimeric form the active site is localized close to the subunit interface, in a cleft between the domains.
Most of the strictly conserved amino-acid residues are located on the dimer surface and nearly all are clustered close to the active site (Capitani et al., 1999). The crystallographic data imply that the large domain distinguished in the three-dimensional structure of ACS from apple and tomato contains the majority of the conserved regions (region 2-6). The crystal structures of ACC synthase from tomato and apple are very similar.

\section{EVOLUTIONARY RELATIONSHIPS OF ACC SYNTHASE}

The lysine residue forming the internal aldimine with $\mathrm{C}^{\prime}$ ' of PLP is absolutely conserved among all members of the PLP-dependent superfamily. The enzymes which use the pyridoxal moiety of PLP for catalysis have been classified into four structural fold types (29 enzyme structures deposited in the Protein Data Bank). The four types of PLP-dependent enzymes do not have a common structure fold but a limited conservation of the PLP binding residues. The type I fold consists of a large $\alpha \beta \alpha$ sandwich domain and a small $\alpha \beta$ domain. Type I has been divided into subgroups on the basis of conformational changes upon the binding of substrate or inhibitors: subgroup Ia - large conformational changes, subgroup Ib - absence of conformational changes. The crystal structure of LE-ACS2 from tomato suggests that this enzyme belongs to subgroup Ib rather than to Ia (Huai et al., 2001).

The PLP-dependent enzymes are found in four out of the six EC classes of enzymes but sequence and structural alignments indicate that ACC synthases are most closely evolutionarily related to the subgroup I of aminotransferases which includes aspartate, alanine, histidinolphosphate, tyrosine and phenylalanine aminotransferases (Alexander et al., 1994; Mehta \& Christen, 1994; Feng et al., 2000).

There are only 12 amino-acid residues completely conserved among various amino- 
transferases. All but one of them are present in appropriate places in all ACC synthases (Rottmann et al., 1991; Theologis, 1992). Dimerization is necessary for the catalytic activity of aminotransferases. Aspartate aminotransferase has two active sites and each of them is formed by the juxtaposition of essential residues from both subunits (Feng et al., 2000).

The closest three-dimensional structural homologues of apple ACS are Thermus thermophilus aspartate and Trypanosoma cruzi tyrosine aminotransferases, but Bacillus sp. aspartate and rat tyrosine aminotransferases are the most similar ones to apple ACS in the primary structure (Feng et al., 2000; Capitani et al., 1999). Other structures of the PLP-dependent enzymes that align well with apple ACS three-dimensional structure include pig cytosolic aspartate aminotransferase, and $E$. coli $\beta$-cystathionase and 8-amino-7-oxonanoate synthase.

ACC synthase has been found and its function is known only in higher plants and in the fungus Penicillium citrinum. Possible homologues of plant ACS were found in the human and pufferfish (Fugu rubripes) genomes. Transcripts of these genes were detected in human and $F$. rubripes cDNA libraries. The function of the encoded proteins have not been resolved yet. The human homologue of ACS has bound PLP but does not convert SAM to ACC. This homologue is capable of deamination of L-VG (Feng et al., 2000; Feng \& Kirsch, 2000; Peixoto et al., 2000; Koch et al., 2001).

Members of the PLP-dependent family are characterized by significant conservation of the PLP binding residues. It is not known whether they represent convergent evolution or evolved divergently from a common origin.

A phylogenetic tree of ACS isoenzymes from various plant species constructed on the basis of amino-acid sequence identity implies that the ACS gene divergence had taken place before monocots and dicots separation. ACS genes were then divided into three main groups (Liang et al., 1992; Destefano-Beltran et al., 1995; Subramaniam et al., 1996; Trebitsh et al., 1997). Expression of each of these groups is induced by similar stimuli. The multigene family of ACS reflects the evolution of a set of polypeptides which catalyse the rate-limiting step in ethylene synthesis in various plant tissues during plant growth, development or stress.

\section{R E F E R E N C E S}

Abel S, Nguyen MD, Chow W, Theologis A. (1995) ACS4, a primary indoleacetic acid-responsive gene encoding 1-aminocyclopropane-1-carboxylate synthase in Arabidopsis thaliana. J Biol Chem.; 270: 19093-9.

Alexander FW, Sandmeier E, Mehta PK, Christen P. (1994) Evolutionary relationships among pyridoxal 5 '-phosphate-dependent enzymes. Eur J Biochem.; 219: 953-60.

Arteca JM, Arteca RN. (1999) A multi-responsive gene encoding 1-aminocyclopropane-1-carboxylate synthase (ACS6) in Arabidopsis leaves. Plant Mol Biol.; 39: 209-19.

Avni A, Bailey BA, Mattoo AK, Anderson JD. (1994) Induction of ethylene biosynthesis in Nicotiana tabacum by a Trichoderma viridae xylanase is correlated to the accumulation of 1-aminocyclopropane-1-carboxylic acid synthase and ACC oxidase transcripts. Plant Physiol.; 106: 1049-55.

Barry CS, LLop-Tous MI, Grierson D. (2000) The regulation of 1-aminocyclopropane-1-carboxylic acid synthase gene expression during the transsmition from system 1 to system 2 ethylene synthesis in tomato. Plant Physiol.; 123: 979-86.

Bekman EP, Saibo NJM, Di Cataldo A, Regalado AP, Ricardo CP, Rodrigues-Pousada C. (2000) Differential expression of four genes encoding 1-aminocyclopropane-1-carboxylate synthase in Lupinus albus during germination, and in response to indole-3-acetic acid and wounding. Planta.; 211; 663-72.

Botella JR, Arteca JM, Schlagnhaufer CD, Arteca RN, Phillips AT. (1992) Identification 
and characterization of a full-length cDNA encoding for an auxin-induced

1-aminocyclopropane-1-carboxylate synthase from etiolated mung bean hypocotyl segments and expression its mRNA in response to indole-3-acetic acid. Plant Mol Biol.; 20: 425-36.

Botha M-L, Whitehead CS. (1992) The effect of polyamines on ethylene synthesis during normal and pollination-induced senescence of $\mathrm{Pe}$ tunia hybrida flowers. Planta.; 188: 478-83.

Capitani G, Hohenester E, Feng L, Storici P, Kirsch JF, Jansonius JN. (1999) Structure of 1-aminocyclopropane-1-carboxylate synthase, a key enzyme in the biosynthesis of the plant hormone ethylene. J Mol Biol.; 294: 745-56.

Casas JL, Garcia-Canovas F, Tudela J, Acosta M. (1993) A kinetic study of simultaneous suicide inactivation and irreversible inhibition of an enzyme. Application to 1-aminocyclopropane-1-carboxylate (ACC) synthase inactivation by its substrate S-adenosylmethionine. J Enzyme Inhib.; 7: $1-14$.

Clark DG, Richards C, Hilioti Z, Lind-Iversen S, Brown K. (1997) Effect of pollination on accumulation of ACC synthase and ACC oxidase transcripts, ethylene production and flower petal abscission in geranium. Plant Mol Biol.; 34: 855-65.

Destefano-Beltran LJC, Caeneghem WV, Gielen J, Richard L, Van Montagu M, Van Der Straeten D. (1995) Characterization of three members of the ACC synthase gene family in Solanum tuberosum. Mol Gen Genet.; 246: 496-508.

Eliot AC, Kirsch JF. (2002) Modulation of the internal aldimine $\mathrm{p} K(\mathrm{a})$ 's of 1-aminocyclopropane-1-carboxylate synthase and aspartate aminotransferase by specific active site residues. Biochemistry.; 41: 3836-42.

Feng 1, Kirsch JF. (2000) L-Vinylglycine is an alternative substrate as well as mechanism-based inhibitor of 1-aminocyclopropane-1-carboxylate synthase. Biochemistry.; 39: 2436-44.

Feng L, Geck MK, Eliot AC, Kirsch JF. (2000) Aminotransferase activity and bioinformatic analysis of 1-aminocyclopropane-1-carboxylate synthase. Biochemistry.; 39: 15242-9.

Hohenester E, White MF, Kirsch JF, Jansonius JN. (1994) Crystallization and preliminary X-ray analysis of recombinant 1-aminocyclopropane-1-carboxylate synthase from apple. $J$ Mol Biol.; 243: 947-9.

Huai Q, Xia Y, Chen Y, Callahan B, Li N, Ke H. (2001) Crystal structures of ACC synthase in complex with AVG: a new insight into catalytic mechanism. J Biol Chem.; 276: 38210-6.

Huang P-L, Parks JE, Rottmann WH, Theologis A. (1991) Two genes encoding 1-aminocyclopropane-1-carboxylate synthase in zucchini (Cucurbita pepo) are clustered and similar but differentially regulated. Proc. Natl. Acad. Sci. U S A.; 88: 7021-5.

Huxtable S, Zhou H, Wong S, Li N. (1998)

Renaturation of

1-aminocyclopropane-1-carboxylate synthase expressed in Escherichia coli in the form of inclusion bodies into a dimeric and catalytically active enzyme. Prot Expr Purif.; 12: 305-14.

Hyodo H, Tanaka K. (1986) Inhibition of 1-aminocyclopropane-1-carboxylic acid synthase activity by polyamines, their related compounds and metabolites of S-adenosylmethionine. Plant Cell Physiol.; 27: 391-8.

Icekson I, Apelbaum A. (1983) Antifungal antibiotics and SIBA inhibit 1-aminocyclopropane-1-carboxylic acid synthase activity. Biochem Biophys Res Commun.; 113: 586-91.

Ishiki Y, Oda A, Yaegashi Y, Orihara Y, Arai T, Hirabayashi T, Nakagawa H, Sato T. (2000) Cloning of an auxin-responsive 1-aminocyclopropane-1-carboxylate synthase gene from melon and the expression of ACS genes in etiolated melon seedlings and melon fruits. Plant Sci.; 159: 173-81.

Jia Y-J, Kakuta Y, Sugawara M, Igarashi T, Oki N, Kisaki M, Shoji T, Kanetuna Y, Horita T, Matsui H, Honma M. (1999) Synthesis and degradation of 1-aminocyclopro- 
pane-1-carboxylic acid by Penicillium citrinum . Biosci Biotech Biochem.; 63: 542-9.

Kakuta Y, Igarashi T, Murakami T, Ito H, Matsui H, Honma M. (2000)

1-Aminocyclopropane-1-carboxylate synthase of Penicillium primary structure and expression in Escherichia coli and S. cerevisiae. Biosci Biotech Biochem.; 65: 1511-8.

Kamachi S, Sekimoto H, Kondo N, Sakai S. (1997) Cloning of a cDNA for a 1-aminocyclopropane-1-carboxylate synthase that is expressed during development of female flowers at the apices of Cucumis sativus. Plant Cell Physiol.; 38: 1197-206.

Kathiseran A, Nagarathna KC, Moloney MM, Reid DM, Chinnappa CC. (1998) Differential regulation of

1-aminocyclopropane-1-carboxylate synthase gene family and its role in phenotypic plasticity in Stellaria longipes. Plant Mol Biol.; 36: 265-74.

Kato M, Hayakawa Y, Hyodo H, Ikoma Y, Yano M. (2000) Wound-induced ethylene synthesis and expression and formation of 1-aminocyclopropane-1-carboxylate synthase, ACC oxidase, phenylalanine ammonia-lyase, and peroxidase in wounded mesocarp tissue of Cucurbitsa maxima. Plant Cell Physiol.; 41: 440-7.

Kende H. (1993) Ethylene biosynthesis. Annu Rev Plant Physiol Plant Mol Biol.; 44: 283-307.

Klee HJ, Hayford MB, Kretzmer KA, Barry GF, Kishore GM. (1991) Control of ethylene synthesis by expression of a bacterial enzyme in transgenic tomato plants. Plant Cell.; 3: 1187-93.

Koch KA, Capitani G, Gruetter MG, Kirsch JF. (2001) The human cDNA for a homologue of the plant enzyme

1-aminocyclopropane-1-carboxylate synthase encodes a protein activity. Gene.; 272 : $75-84$.

Lelievre J-M, Tichit L, Dao P, Fillion L, Nam Y-W, Pech J-C, Latche A. (1997) Effects of chilling on the expression of ethylene biosynthetic genes in Passe-Crassane pear fruits. Plant Mol Biol.; 33: 847-55.
Li N, Mattoo A. (1994) Deletion of the carboxyl-terminal region of 1-aminocyclopropane-1-carboxylic acid synthase, a key protein in the biosynthesis of ethylene, results in catalytically hyperactive, monomeric enzyme. J Biol Chem.; 269: 6908-17.

Li N, Jiang XN, Cai GP, Yang SF. (1996a) A novel bifunctional fusion enzyme catalyzing ethylene synthesis via 1-aminocyclopropane-1-carboxylic acid. J Biol Chem.; 271: 25738-41.

Li N, Huxtable S, Yang SF, Kung SD. (1996b) Effects of N-terminal deletions on 1-aminocyclopropane-1-carboxylate synthase activity. FEBS Lett.; 378: 286-90.

Li Y, Feng L, Kirsch JF. (1997) Kinetic and spectroscopic investigations of wild-type and mutant forms of apple 1-aminocyclopropane-1-carboxylate synthase. Biochemistry.; 36: 15477-88.

Liang X, Abel S, Keller JA, Shen NF, Theologis A. (1992) The 1-aminocyclopropane-1-carboxylate synthase gene family of Arabidopsis thaliana. Proc Natl Acad Sci U S A.; 89: 11046-50.

Liang X, Oono Y, Shen NF, Kohler C, Li K, Scolnik PA, Theologis A. (1995) Characterization of two members (ACS1 and ACS3) of the 1-aminocyclopropane-1-carboxylate synthase gene family of Arabidopsis thaliana. Gene.; 167: 17-24.

Liang X, Shen NF, Theologis A. (1996) Li ${ }^{+}$-regulated 1-aminocyclopropane-1-carboxylate synthase gene expression in Arabidopsis thaliana. Plant J.; 10: 1027-36.

Lincoln JE, Campbell AD, Oetiker J, Rottmann WH, Oeller PW, Shen NF, Theologis A. (1993) LE-ACS4, a fruit ripening and wound-induced 1-aminocyclopropane-carboxylate synthase gene of tomato (Lycopersicon esculentum). $J$ Biol Chem.; 268: 19422-30.

Llop-Tous I, Barry CS, Grierson D. (2000) Regulation of ethylene biosynthesis in response to pollination in tomato flowers. Plant Physiol.; 123: $971-8$. 
Mattoo AK, Anderson JD, Chalutz E, Lieberman M. (1979) Influence of enol ether amino acids, inhibitors of ethylene biosynthesis, on aminoacyl transfer RNA synthetases and protein synthesis. Plant Physiol.; 64: 289-92.

McCarthy DL, Capitani G, Feng L, Gruetter MG, Kirsch JF. (2001) Glutamate 47 in 1-aminocyclopropane-1-carboxylate synthase is a major specifity determinant. Biochemistry.; 40: 12276-84.

Mehta PK, Christen P. (1994) Homology of 1-aminocyclopropane-1-carboxylate synthase, 8-amino-7-oxonanoate synthase, 2-amino-6-caprolactam racemase, 2,2-dialkylglycine decarboxylase, glutamate-1-semialdehyde 2,1-aminomutase and isopenicillin- $N$-epimerase with aminotransferases. Biochem Biophys Res Commun.;198: 138-43.

Miki T, Yamamoto M, Nakagawa H, Ogura N, Mori H, Imaseki H, Sato T. (1995) Nucleotide sequence of a cDNA for 1-aminocyclopropane-1-carboxylate synthase from melon fruits. Plant Physiol.; 107: 297-8.

Miura GA, Chiang PK. (1985) A radioisotope assay for 1-aminocyclopropane-1-carboxylic acid synthase: S-adenosylhomocysteine analogs as inhibitors of the enzyme involved in plant senescence. Anal Biochem.; 247: 217-21.

Nakajima N, Nakagawa N, Imaseki H. (1988) Molecular size of wound-induced 1-aminocyclopropane-1-carboxylate synthase from Cucurbita maxima Duch. and change of translatable mRNA of the enzyme after wounding. Plant Cell Physiol.; 29: 989-98.

Nakatsuka A, Murachi S, Okunishi H, Shiomi S, Nakano R, Kubo Y, Inaba A. (1998) Differential expression and internal feedback regulation of 1-aminocyclopropane-1-carboxylate synthase, ACC oxidase, and ethylene receptor genes in tomato fruit during development and ripening. Plant Physiol.; 118: 1295-305.

Oetiker JH, Olson DC, Shiu OY, Yang SF. (1997) Differential induction of seven 1-aminocyclopropane-1-carboxylate synthase genes by elicitor in suspension cultures of to- mato (Lycopersicon esculentum). Plant Mol Biol.; 34: 275-86.

Olson DC, Oetiker JH, Yang SF. (1995) Analysis of LE-ACS3, a

1-aminocyclopropane-1-carboxylic acid synthase gene expressed during flooding in the roots of tomato plants. J Biol Chem.; 270: 14056-61.

Park KY, Drory A, Woodson WR. (1992) Molecular cloning of an 1-aminocyclopropane-1-carboxylate synthase from senescing carnation flower petals. Plant Mol Biol.; 18: 377-86.

Peixoto BR, Mikawa Y, Brenner S. (2001) Characterization of the recombinase activating gene- 1 and 2 locus in Japanese pufferfish, Fugu rubripes. Gene.; 246: 275-83.

Pogson BJ, Downs CG, Davies KM, Morris SC. (1995) Nucleotide sequence of a cDNA clone encoding 1-aminocyclopropane-1-carboxylic acid synthase from Broccoli. Plant Physiol.; 108: $857-8$.

Rottmann WH, Peter GF, Oeller PW, Keller JA, Shen NF, Nagy BP, Taylor LP, Campbell AD, Theologis A. (1991)

1-Aminocyclopropane-1-carboxylate synthase in tomato is encoded by a multigene family whose transcription is induced during fruit and floral senescence. J Mol Biol.; 222: 937-61.

Satoh S, Esashi Y. (1986) Inactivation of 1-aminocyclopropane-1-carboxylic acid synthase of etiolated mung bean hypocotyl segments by its substrate, $S$-adenosyl-L-methionine. Plant Cell Physiol.; 91: 1036-9.

Satoh S, Mori H, Imaseki H. (1993) Monomeric and dimeric forms and the mechanism-based inactivation of 1-aminocyclopropane-1-carboxylate synthase. Plant Cell Physiol.; 34: 753-60.

Schlagnhaufer CD, Arteca RN, Pell EJ. (1997) Sequential expression of two 1-aminocyclopropane-1-carboxylate synthase genes in response to biotic and abiotic stresses in potato leaves. Plant Mol Biol.; 35: 683-8. 
Shiu OY, Oetiker JH, Yip WK, Yang SF. (1998) The promoter of $L E-A C S 7$, an early flooding-induced 1-aminocyclopropane-1-carboxylate synthase gene of the tomato, is tagged by a Sol3 transposon. Proc Natl Acad Sci U S A.; 95: 9796-801.

Spanu P, Boller T, Kende H. (1993) Differential accumulation of transcripts of 1-aminocyclopropane-1-carboxylate synthase genes in tomato plants infected with Phytophthora infectans and in elicitor-treated tomato cell suspension. J Plant Physiol.; 141: $557-62$.

Subramaniam K, Abbo S, Ueng PP. (1996) Isolation of two differentially expressed wheat ACC synthase cDNA and the characterization of one of their genes with root-predominant expression. Plant Mol Biol.; 31: 1009-20.

Tarun AS, Theologis A. (1998) Complementation analysis of mutants of 1-aminocyclopropane-1-carboxylate synthase reveals the enzyme is a dimer with shared active sites. J Biol Chem.; 273: 12509-14.

Tarun AS, Lee JS, Theologis A. (1998) Random mutagenesis of 1-aminocyclopropane-1-carboxylate synthase: A key enzyme in ethylene biosynthesis. Proc Natl Acad Sci U S A.; 95: 9796-801.

Tatsuki M, Mori H. (1999) Rapid and transient expression of 1-aminocyclopropane-1-carboxylate synthase isogenes by touch and wound stimuli in tomato. Plant Cell Physiol.; 40: 709-15.

Theologis A. (1992) One rotten apple spoils the whole bushel: the role of ethylene in fruit ripening. Cell.; 70: 181-4.

Theologis A, Zarembinski TI, Oeller PW, Liang X, Abel S. (1992) Modification of fruit ripening by suppressing gene expression. Plant Physiol.; 100: 549-51.

Theologis A, Oeller PW, Wong L-M, Rottmann WH, Gantz DM. (1993) Use of tomato mutant constructed with reverse genetics to study fruit ripening, a complex developmental process. Dev Genet.; 14: 282-95.

Trebitsh T, Staub JE, O’Neill SD. (1997) Identification of a 1-aminocyclopropane-1-carboxylic acid synthase gene linked to the Female (F) locus that enhances female sex expression in cucumber. Plant Physiol.; 113: 987-95.

Tuomainen J, Betz C, Kangasjarvi J, Ernst D, Yin Z-H, Langebartels C, Sanderman H. (1997) Ozone induction of ethylene emission in tomato plants: regulation by differential accumulation of transcripts for the biosynthetic enzymes. Plant J.; 12: 1151-62.

Wang T-W, Arteca RN. (1995) Identification and characterization of cDNAs encoding ethylene biosynthetic enzymes from Pelargonium $x$ hortorum cv Snow Mass leaves. Plant Physiol.; 109: 627-36.

White MF, Vasques J, Yang SF, Kirsch JF. (1994) Expression of apple 1-aminocyclopropane-1-carboxylate synthase in Escherichia coli: kinetic characterization of wild-type and active-site mutant forms. Proc Natl Acad Sci U S A.; 91: 12428-32.

Wong WS, Ning W, Xu PL, Kung SD, Yang SF, Li N. (1999) Identification of two chilling-regulated 1-aminocyclopropane-1-carboxylate synthase genes from citrus fruit. Plant Mol Biol.; 41: 587-600.

Yang SF, Hoffman NE. (1984) Ethylene biosynthesis and its regulation in higher plants. Annu Rev Plant Physiol.; 35: 155-89.

Yasuta T, Satoh S, Minamisawa K. (1999) New assay for rhizobitoxine based on inhibition of 1-aminocyclopropane-1-carboxylate synthase. Appl Env Microb.; 65: 849-52.

Yip W-K, Yang SF. (1988) Cyanide metabolism in relation to ethylene production in plant tissues. Plant Physiol.; 88: 473-6.

Zarembinski TI, Theologis A. (1993) Anaerobiosis and plant growth hormones induce two genes encoding 1-aminocyclopropane-1-carboxylate synthase in rice. Mol Biol Cell.; 4: 363-73.

Zarembinski TI, Theologis A. (1994) Ethylene biosynthesis and action: case of conservation. Plant Mol Biol.; 26: 1579-97.

Zarembinski TI, Theologis A. (1997) Expression characteristics of OS-ASC1 and OS-ACS2, two members of the 1-aminocyclopropane-1-carboxylate synthase gene family in 
rice during partial submergence. Plant Mol Biol.; 33: 71-7.

Zhou H, Wang HW, Zhu K, Sui SF, Xu P, Yang SF, Li N. (1999) The multiple roles of con- served arginine 286 of 1-aminocyclopropane-1-carboxylate synthase. Coenzyme binding, substrate binding, and beyond. Plant Physiol.; 121: 913-9. 